\title{
Power sizing factor design of central inverter PV grid-connected systems: a simulation approach.
}

\author{
G. Velasco, R. Piqué, F. Guinjoan, F. Casellas and J. de la Hoz
}

\begin{abstract}
This paper presents a simulation approach which can help in the preliminary power sizing design of a gridconnected PV system based on a single inverter configuration. Given a nominal peak power of the PV array, this simulation procedure leads to the $P V$ inverter maximum rated power which maximizes the yearly injected energy to the grid.
\end{abstract}

\section{INTRODUCTION}

PHOTOVOLTAIC energy generation provides several advantages such as being harmless for the environment and renewable. Furthermore, grid-connected PV energy generation represents a renewable energy growing alternative that is becoming more competitive due to the new favourable governmental laws and policies as recently introduced.

The Plant Oriented (PO) configuration is one of the most prevailing PV grid-connected system's architectures due to its simplicity and low cost per $k W_{P}$ [1], and assumes a single solar array referred as Photovoltaic Generator (PVG) which is linked to the grid through a single central inverter. This inverter is in charge to extract at any time the maximum power from the PVG and to properly transfer this power to the grid.

At a preliminary stage, the design of such systems firstly addresses the choice of both the PVG peak power given in Standard Test Conditions ${ }^{1}$ (STC) $\left(P_{P V G, S T C}\right)$ and the maximum rated power of the central inverter $\left(P_{\text {INVMAX }}\right)$. The ratio between these two values is known as the "Sizing Factor" $(S F)$ which constitutes one of the main design parameters of the PV installation and is defined as:

$$
P_{I N V M A X}=S F \cdot P_{P V G, S T C}
$$

The standard design procedure firstly determines the peak power of the PVG $\left(P_{P V G, S T C}\right)$ taking into account several conditioning factors such as the available surface at the PV site, location with no shadowing patterns (if possible), and cost investments. Subsequently, the inverter maximum rated power would be chosen to maximize the yearly energy delivered to the grid. In other words, the design would determine the optimum sizing factor value $\left(S F_{O P T}\right)$, i.e. the value which maximizes the yearly injected energy.

However, most of the designs ignore this procedure and heuristically set the SF to values lying between $60 \%$ and $80 \%$ [2-3], i.e. under sizing the inverter maximum power with respect to the peak power of the PVG. This extended practice just takes qualitatively into account that the PVG operates most of the time below the irradiance level and above the temperature given by the STC.
Nevertheless, deriving the optimum value of the sizing factor is not a trivial task since the yearly produced energy depends on the power processing features of the elements involved in the PV power conversion chain, namely:

- Irradiance and temperature evolution at the PV installation site.

- Characteristics of the PVG: PV cell material, PV installation mounting type ...

- DC wiring losses.

- Inverter electrical characteristics: Maximum power, efficiency curve...

In order to avoid an heuristic power sizing practice, the work here reported presents a simulation approach to estimate the optimum Sizing Factor value, which is based on contrasted models found in the specialized literature of the power conversion chain. In this regard, it must be pointed out that the simulation procedure here reported assumes:

- An uninterrupted operation of the PV system neglecting the reliability and durability influence of the components of the PV conversion chain as well as the possible inverter disconnections due to $\mathrm{AC}$ side failures [4].

- An uniform distribution of the irradiance and ambient temperature levels in the PVG (i.e. shadowing due to surrounding obstacles is not considered).

The paper content extends the results of previous works [5] and is organized as follows: Section II describes the models used for all the elements involved in the PV system conversion chain. Section III focuses on the simulation procedure leading to the sizing factor optimal value maximizing the yearly delivered energy. This simulation procedure is applied in section IV for different PV site locations and inverter efficiencies to evaluate their influence on the sizing factor optimal value.

Finally, section $\mathrm{V}$ draws the conclusions with the aim of helping the preliminary power sizing design of PV gridconnected systems based on a single central inverter.

\section{PV SYSTEM MODELING}

The following description of the simulation-oriented models refers to the variables of the PV power conversion chain depicted in Fig.1:

${ }^{1}$ Irradiance of $1000 \mathrm{~W} / \mathrm{m}^{2}, \mathrm{AM} 1.5$ solar spectrum, temperature of $25^{\circ} \mathrm{C}$ 


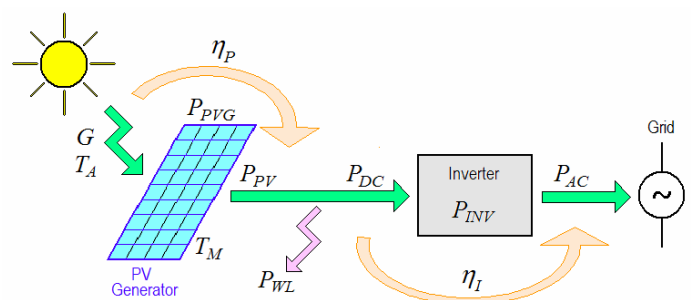

Fig. 1. Elements of the PV power conversion chain.

\section{A) Solar Irradiance and temperature}

The available power at PVG output $\left(P_{P V}\right)$ depends, among others, on the solar irradiance and temperature at the latitude, longitude and altitude of the PV installation location. Correspondingly, the $S F$ value will also exhibit this dependence and requires an estimation of these environmental variables for different PV sites. This estimation is commonly based on averaged data over a fixed period whose values are still controversial as regards the effects of transient shades (due to clouds) in the irradiance estimation. Even that the output power drops when the PVG operates under shade conditions and these peak irradiance levels would lead to different $S F$ values, both the irradiance level and the time at which they occur are unpredictable as long as they depend on environmental random factors. As a compromise, this work has considered the 15 minutes average solar irradiance and temperature data, available at the PVGIS ${ }^{2}$ database, for the capitals of the 27 European Union countries (see Fig. 2). These data correspond to the daily evolution of ambient temperature $\left(T_{A}\right)$ and incident irradiance $(G)$ on a flat surface with optimal yearly tilt.

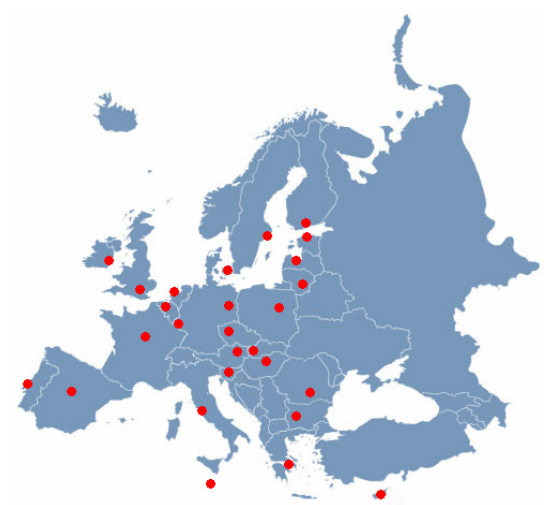

Fig. 2. PV site locations considered in the analysis.

\section{B) PV generator model}

The DC power at the PVG output $\left(P_{P V}\right)$ depends on the incident irradiance $(G)$, the PVG surface $\left(S_{P V G}\right)$ and its efficiency $\left(\eta_{P}\right)$ and is given by [6]:

$$
P_{P V}=\eta_{P} \cdot G \cdot S_{P V G}
$$

The PVG efficiency is, in turn, dependent on the PV modules operating temperature $\left(T_{M}\right)$ and the $\mathrm{PV}$ cell material and can be expressed as:

${ }^{2}$ PVGIS: Photovoltaic Geographical Information System. Online available at: http://re.jrc.ec.europa.eu/pvgis/

$$
\eta_{P}=\eta_{R} \cdot\left[1-\beta_{P}\left(T_{M}-T_{R}\right)\right]
$$

where $\eta_{R}(\%)$ is the PVG efficiency at the reference temperature, $\beta_{P}\left(\% /{ }^{\circ} \mathrm{C}\right)$, is the thermal efficiency coefficient of the PVG material being $T_{R}$ the reference temperature $\left(25^{\circ} \mathrm{C}\right)$. The values of $\eta_{R}$ and $\beta_{P}$ depend on the semiconductor material. For instance for $\mathrm{m}-\mathrm{Si} \mathrm{PV}$ cells, these values are $\eta_{R}=13 \%$ and $\beta_{P}=0.5 \% /{ }^{\circ} \mathrm{C}$ [6].

On the other hand, the PVG operating temperature $\left(T_{M}\right)$ is related to the ambient one $\left(T_{A}\right)$ as given in [7-8], namely:

$$
T_{M}=T_{A}+k \cdot G
$$

where $k\left({ }^{\circ} \mathrm{C} \cdot \mathrm{m}^{2} / \mathrm{W}\right)$ is the PVG thermal coefficient according to mounting type (Ross coefficient). The value of $k$ is related with the PVG ventilation capability (natural ventilation by convection or forced ventilation by wind or airflows) and thereby depends on the PV installation mounting type. For instance, the typical value of $k$ for a well cooled installation is $k=0.02$ [7-8]. In summary, the PVG model relating the output power and the irradiance can be obtained by combining Eqs. (2 - 4), namely:

$$
P_{P V}=\eta_{R} \cdot\left[1-\beta_{P} \cdot\left(T_{A}+k \cdot G-T_{R}\right)\right] \cdot G \cdot S_{P V G}
$$

The parameters $\eta_{R}, \quad \beta_{P}$ and $k$ highlight the model dependence on the PV cell material and the PV installation mounting type respectively. Consequently, the sizing ratio dependence with these parameters can be evaluated.

C) DC losses on the PV generator

If $P_{W L}$ stands for the power losses on the PVG wires, the DC power at the inverter input, $P_{D C}$ is given by:

$$
P_{D C}=P_{P V}-P_{W L}
$$

On the other hand, DC losses for PV systems based on $m$ Si modules are usually estimated around $1 \%$ of the nominal power of the PV system [9]. Accordingly, Eq. (6) can be rewritten as:

$$
P_{D C_{-} m-S i}=P_{P V}-\frac{1}{100} \cdot P_{P V, S T C_{-} m-S i}
$$

Alternatively, an accurate estimation of these losses for any PV cell material can be found in the work of Perez et al. [10] through the following expression:

$$
P_{W L}=\alpha \cdot \rho_{W} \cdot \frac{L_{W}}{S_{W}} \cdot I_{M, S T C}^{2} \cdot H_{d a}^{2}
$$

where $\alpha$ is a constant coefficient given in $\left(\mathrm{m}^{2} / \mathrm{kWh}\right)^{2}, \rho_{W}, L_{W}$ and $S_{W}$ are the resistivity, the length and the cross-section area of wires respectively, $I_{M, S T C}$ stands for the current at MPP in STC and $H_{d a}^{2}$ (given in $\mathrm{kWh} / \mathrm{m}^{2}$ ) corresponds to the yearly mean daily irradiation at the PVG surface. Since both $\alpha$ and $I_{M, S T C}$ values depend, among others, on the semiconductor type, this expression can be used for any PV cell material. Further simplifications of this expression can be made but are beyond the scope of the paper. 


\section{D) Inverter model}

Provided that the inverter input power does not exceed the maximum inverter rated power, noted as $P_{I N V M A X}$, the available power at the inverter output $\left(\mathrm{P}_{A C}\right)$ is given by:

$$
P_{A C}=\eta_{I} \cdot P_{D C}
$$

where $\eta_{I}$ stands for the inverter efficiency, which can be modeled as [11-12]:

$$
\eta_{I}=\frac{p}{k_{0}+\left(1+k_{1}\right) \cdot p+k_{2} \cdot p^{2}} \quad ; \quad p=\frac{P_{A C}}{P_{\text {INVMAX }}}
$$

where $k_{0}$ stands for the losses coefficient at no load, being $k_{l}$ - $k_{2}$ linear and quadratic current losses coefficients. Replacing (9) into (10) and solving for $P_{A C}$, leads to:

$$
P_{A C}=\frac{-\left(1+k_{1}\right)+\sqrt{\left(1+k_{1}\right)^{2}-4 \cdot k_{2} \cdot\left(k_{0}-\frac{P_{D C}}{P_{I N V M A X}}\right)}}{2 \cdot \frac{k_{2}}{P_{I N V M A X}}}
$$

For input power ranges higher than the inverter maximum rated power, this work assumes the operating mode suggested in [12] to avoid power delivery interruptions, i.e. the inverter's control limits the input power to its maximum value until the overload conditions are no longer present. Therefore, in this case:

$$
P_{A C}=P_{I N V M A X}
$$

In summary, given the input power $P_{D C}$ and the inverter maximum power $P_{I N V M A X}$, the AC output power can be computed from Eqs. (11-12). The losses coefficients values adopted in this work are listed in Table I from an exhaustive laboratory test of a large number of PV grid-connected inverters reported in [12] which classifies the inverter's efficiency into two categories: low and high efficiency.

Table I: Values of losses coefficients for low and high efficiency

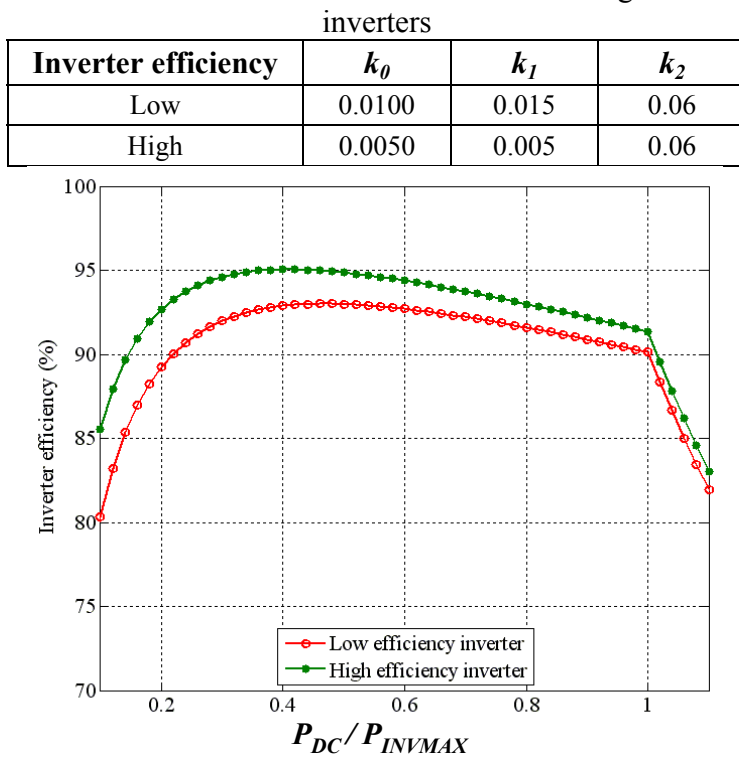

Fig. 3. Efficiencies of low and high efficiency inverters.
The corresponding efficiencies are plotted in Fig. 3 in terms of $P_{D C} / P_{I N V M A X}$. As it can be seen, the maximum efficiency value is nearly $95 \%$ for the high efficiency inverter and $93 \%$ for the low efficiency one. Furthermore, the figure also shows the better performance of the high efficiency inverter under partial load operation.

\section{SiMULATION PROCEDURE}

Fig. 4 shows the block-diagram of the simulation procedure. The simulation computes the yearly injected energy for different Sizing Factor values by varying the maximum inverter rated power, $P_{\text {INVMAX }}$ (see eq.(1)). Subsequently the value of $S F_{O P T}$ which maximizes the yearly injected energy can be deduced.

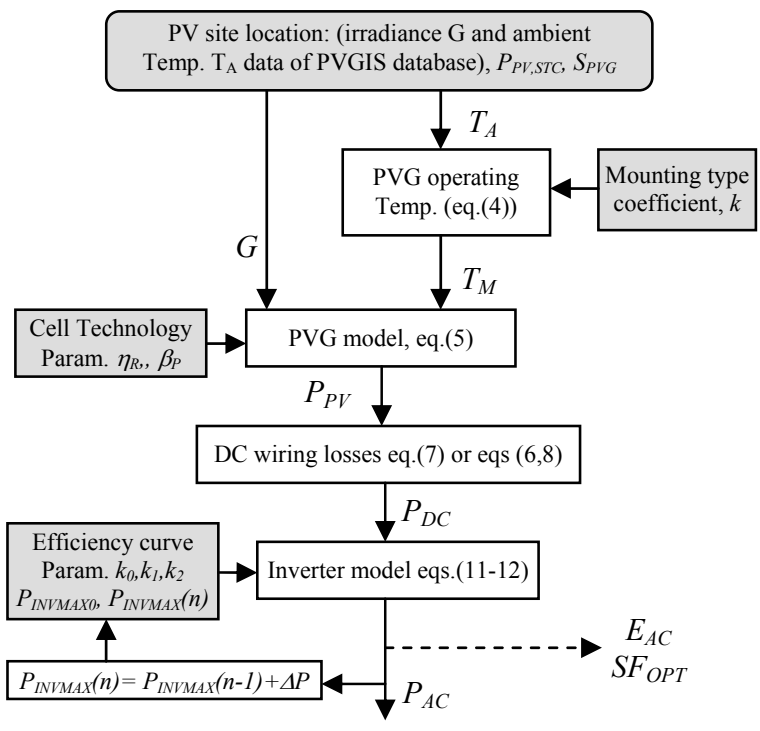

Fig. 4. Block-diagram of the simulation procedure.

The simulation requires from the user the initial setting of the following parameters (grey blocks): PV site location (i.e. irradiance and ambient temperature data available at the PVGIS database), the nominal peak power of the PVG $\left(P_{P V G, S T C}\right)$ and its surface $S_{P V G}$, the installation mounting type (i.e. the parameter $k$ involved in (4)), the PVG cell technology (i.e. parameters $\eta_{R}$ and $\beta_{P}$ involved in (3)), the inverter efficiency curve (i.e. losses coefficients defined in (11)), and eventually the parameters required by (8) if the PV cells are of different material than $m$-Si. In addition, an initial value of the inverter maximum rated power $P_{\text {INVMAXO }}$ (an initial value of the $S F$ ) is required to start the simulation.

For each of the values of $15 \mathrm{~min}$ average irradiance and ambient temperature available at the PVGIS database over one year, the simulation sequentially computes the available power at the PVG output $\left(P_{P V}\right)$, the DC power at inverter input $\left(P_{D C}\right)$ and the power delivered to the grid $\left(P_{A C}\right)$ from the expressions shown in Fig.4. Subsequently, the yearly injected energy, $E_{A C}$ is computed as:

$$
E_{A C} \approx \Delta t \cdot \sum_{n=1}^{m} P_{A C}(n)
$$


where $\Delta t=15 \mathrm{~min}$ and $m$ is the total number of available samples at the PVGIS database over one year. This procedure is carried out 15 times to range the Sizing Factor from 0 to 1.5 with steps of 0.1 , by varying accordingly the inverter maximum rated power value. From these simulation results, the value of $S F_{O P T}$ maximizing the yearly injected energy can be obtained. In can be noted that different parameter combinations of PV site locations, PV cell materials, inverter efficiencies as well as installation mounting types can be considered in the simulations to evaluate their influence on the sizing ratio optimal value.

\section{IV.SIMULATION RESULTS}

The simulation procedure described in section III has been programmed by means of Matlab ${ }^{\circledR}$ software. The simulation results presented in the following consider different PV site locations and inverter efficiencies. For comparative purposes which will help in the analysis of the $S F$ optimum value, the same PVG of $1 \mathrm{kWp}$ built by $m-S i$ cells and a well-cooled installation mounting type is assumed. In this regard, the parameter values involved in all the simulations are those reported in previous sections

Fig. 5 shows the evolution of the yearly injected energy in $\mathrm{kWh} / \mathrm{kW}_{\mathrm{P}}$, with the sizing ratio for different $\mathrm{PV}$ system locations and for high and low inverter efficiencies.

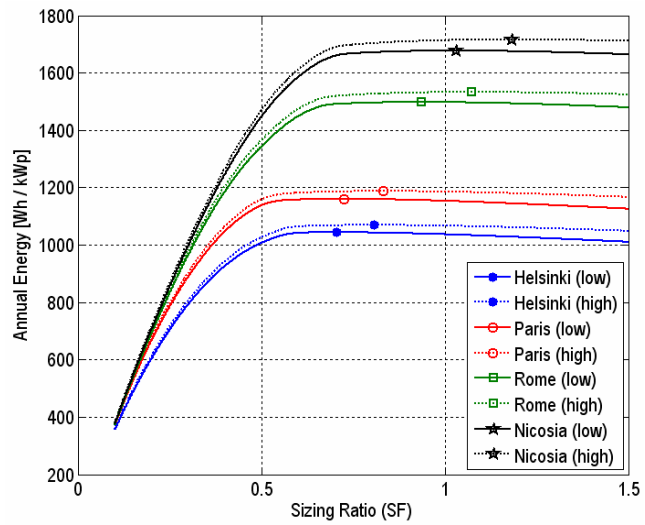

Fig. 5. Annual energy production versus $S F$ value.

This figure shows that each curve presents a single maximum of the yearly energy production (dotted points), which thereby corresponds to the sizing ratio optimal value, $S F_{O P T}$. Furthermore, Fig. 5 also evidences the dependence of the $S F_{O P T}$ value with:

- The location of the PV installation: The $S F_{O P T}$ value increases when the latitude of the PV site location decreases (i.e. the PV site is located closer to the earth equator).

- The inverter type: The $S F_{O P T}$ value increases when the inverter efficiency is higher, but its influence over the annual energy production is small according to the simulations: the use of high efficiency inverters instead of low efficiency ones leads to an increment of $14.6 \%$ of the $S F_{O P T}$ value and of $2.4 \%$ as concerns the yearly energy production.
Furthermore, if high efficiency inverters are sized according to the $S F_{O P T}$ value corresponding to the low efficiency ones, the loss of energy production is only of $0.1 \%$. These results are almost independent of the PV site location.

On the other hand, Fig. 6 shows the evolution of the $S F_{O P T}$ value in terms of all the PV site locations considered in this work and for both inverter efficiencies; the PV site locations have been sorted according to their latitude, i.e. from the city closest to the North Pole (i.e. Helsinki, Lat $60^{\circ} 10^{\prime} \mathrm{N}$ ) to that closest to the Earth equator (i.e. Nicosia, Lat $35^{\circ}$ $\left.10^{\prime} \mathrm{N}\right)$. As it can be seen and confirming the results of Fig. 5, the $S F_{O P T}$ value tends to increase when the latitude of the PV site location decreases. Additionnaly, it is worth noting that the generalized practice of under-sizing the inverter maximum power may result controversial for low-latitude locations such as Rome, Madrid, Lisbon, Athens, Valletta or Nicosia, where the $S F_{O P T}$ is greater than one. Finally Fig. 7 shows the estimation of the annual energy production for all the locations under study when the PV system operates under the $S F_{O P T}$ value. Obviously, the energy production is greater for cities with smaller latitude, and when a high efficiency inverter is adopted.

It must be pointed out that the energy production values obtained in the previous simulations have to be taken with care since they were obtained assuming that the PV generators operate at any time close to ideal conditions: i.e. losses due to irradiance spectrum, mismatching, dust and soiling, shades or AC wiring have not been considered.

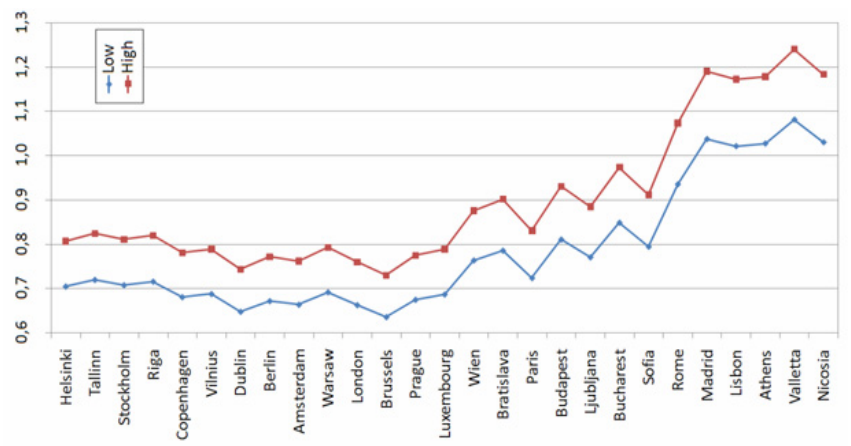

Fig. 6. $S F_{O P T}$ value versus PV system location.

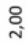

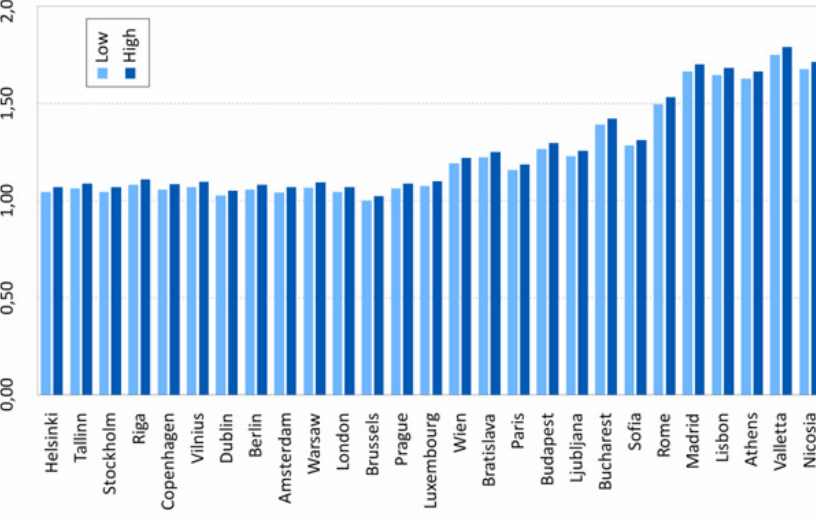

Fig. 7. Annual energy production (in $k W h / k W_{P}$ ) versus PV system location. 


\section{CONCLUSIONS}

This work has presented a simulation procedure leading to the optimum sizing factor value of a PV grid-connected system, which maximizes the yearly energy production. The simulation assumes ideal working conditions and is based on contrasted models found in the literature, which take into account the power generation and processing characteristics of all the elements of the PV conversion chain. On this basis, the influence the PV site location and the inverter efficiency on both the sizing ratio value and the yearly energy production have been analyzed by means of a set of simulations carried out for 27 European PV sites. Based on the simulation results, the following conclusions can be drawn:

- The simulations have shown the existence of a $S F_{O P T}$ single value, which can be greater than one for PV installations located at low latitude sites. Accordingly, for these latitudes the results suggest to oversize the inverter maximum power with respect to the PV generator nominal power, in spite of the under-sizing current practice.

- The $S F_{O P T}$ value increases when the inverter efficiency is higher.

- The $S F_{O P T}$ value is strongly dependent on the irradiance level. Therefore, PV sites with higher average irradiance levels present higher $S F_{O P T}$ values.

- For PV sites with similar levels of average irradiance, the higher $S F_{O P T}$ values correspond to those sites with lower average temperature levels.

- PV sites with a higher $S F_{O P T}$ value are also the PV sites with a higher annual energy production.

It is worth noting that, beside the PV site location and the inverter efficiency, the simulation procedure also supports different parameter combinations of PV cell materials and installation mounting types in order to evaluate their influence on the sizing ratio optimal value.

In conclusion, this simulation approach would help to the preliminary power sizing design task of a central inverter grid-connected PV installation.

\section{ACKNOWLEDGMENTS}

This work has been partially granted by the Spanish Ministry of Science and Innovation and by the EU (FEDER funds) through the grants DPI2009-14713-C03-03 and RUE CSD2009-00046, Consolider-Ingenio 2010 Programme, and was developed at the $U R T E \operatorname{EdePAE}{ }^{3}$ installations, of the EUETIB.

\section{REFERENCES}

[1] M. Meinhardt and G. Cramer. 'Past, Present and Future of grid connected Photovoltaic and Hybrid Power Systems'. IEEE Power Engineering Society Summer Meeting, 2000. Vol. 2, pp. 1283 1288. Seattle, July 2000.

[2] Jayanta Deb Mondol, et al. 'Optimal sizing of array and inverter for grid-connected photovoltaic systems'. Solar Energy. Vol. 80, pp. 1517 - 1539. March 2006.

[3] B. Burger and R. Rüther. 'Inverter sizing of grid-connected photovoltaic systems in the light of local solar resource distribution characteristics and temperature'. Solar Energy. Vol. 80, pp. 32 - 45. March 2006.

[4] G. Petrone, G. Spagnuolo,et al. 'Reliability Issues in Photovoltaic Power Processing Systems'. IEEE Trans. on Industrial Electronics. Vol. 55, no. 7, pp. 2569 - 2580. July 2008.

[5] G. Velasco et al.. 'Inverter Power Sizing Considerations in GridConnected PV Systems'. Proceedings of the 2007 European Conference on Power Electronics and Applications. Pp. 1 - 10. Aalborg, September 2007.

[6] David L. King, Jay A. Kratochvil and William E. Boyson. 'Temperature Coefficients for PV Modules and Arrays: Measurements Methods, Difficulties and Results'. Proceedings of the IEEE $26^{\text {th }}$ PVSEC. Pp. 1183 - 1186. California, October 1997.

[7] T. Nordmann and L. Clavadetscher. 'Understanding Temperature Effects on PV Systems Performance'. Proceedings of the IEEE $3^{\text {rd }}$ WCPEC. Pp. 2243 - 2246. Osaka, May 2003.

[8] E. Skoplaki and J.A. Palyvos. 'Operating Temperature of Photovoltaic Modules: A Survey of Pertinent Correlations'. Renewable Energy. Vol. 34, pp. 23-29. 2009.

[9] T. Oozeki, K. Otani, and K. Kurokawa. 'An Evaluation Method for PV System to Identify System Losses by Means of Utilizing Monitoring Data'. Conference Record of the 2006 IEEE $4^{\text {th }}$ World Conference on Photovoltaic Energy Conversion. Vol. 2, pp. 2319 2322. Hawaii, May 2006.

[10] P. J. Pérez, et al. 'RMS Current of a Photovoltaic Generator in Grid-Connected PV Systems: Definition and Application'. Int. Journal of Photoenergy. Vol. 2008. Article ID 356261.

[11] F. P. Baumgartner, et al. 'Status and Relevance of the DC Voltage Dependency of the Inverter Efficiency'. Proceedings of the $22^{\text {nd }} E U$ PVSEC. Milano, September 2007.

[12] 'Inversores'. Photon - The PV magazine (Spanish edition). Vol. 3/2008, pp. 78 - 93. March 2008. (Article in Spanish).

\footnotetext{
${ }^{3}$ Technology Transfer Unit on Power Electronics and Electric Drives
} 